\title{
Die Frählings-Vegetation
}

\author{
in einem Theile von Algerien.
}

Von Schramm.

Nachstehende Blätter, deren Inhalt so weit es nützlich erschien, einer von mir verfassten Broschure * $)$ entnommen ist, haben den Zweck, ein ungefähres Bild der Frühlings-Flora von Algier zu geben. Ungefähr oder nur annähernd vollständig deshalb, weil ich ohne Absicht auf botanische Forschungen, mithin ganz unvorbereitet, so wie ohne weitere Hilfsmittel ausser dem sehr unvollständigen, und erst späterhin in Algier selbst gekauften M u in by'schen PflanzenVerzeichnisse - Flore de l'Algérie, Paris 1847 - am 26. Februar 1858 den nordafrikanischen Boden betrat, und nur durch die frühzeitige lebhafte Vegelation zur genauen Beobachtung der dortigen Pflanzenwelt angeregt wurde.

Ein jedes fremdartige Ziergewächs; jede groteske Persönlichkeit nahm meine Aufmerksamkeit in Anspruch, wenngleich der untere nahe am Meere gelegene Stadtheil von Algier meist ganz europäisch aussieht und grösstentheils von Europäern bewohnt ist. Ich stieg daselbst mit einem lieben Reisegefährten, dem Lieutenant B a rlels aus Magdeburg, im Hôtel de France ab. Mein kleines einfensteriges Zimmer in der ersten Etage hätte ich mit keinem unter den Linden in Berlin vertauschen mögen, und ich fühlte mich ganz einheimisch und wohl darin. Ich hatte nämlich die freie Aussicht auf den westlichen Theil des ganz nahen Hafens, welcher von der Admiralilät, dem Lenchthurme und der gewaltigen West-Mole begrenzt wird. Handelsfahrzenge von allen Grössen und Fischerbarken beleben ihn.

Gerade aus, also nordwärts, schweift der oft träumerische Blick über das weite Meer, welches hier nach Osten zu die grosse halbmondförmige sichere Bai von Algier bildet, die durch die lieblich bebaumte Küstenstrecke bis zum Cap Matifou abgeschlossen wird. Jenseits dieser, nach Osten zu immer niedriger auslaufenden, Sahelkelte sah ich den mit blendend weissem Schnee bedeckten höheren Theil des Allasgebirges, wahrscheinlich der Dschurdschura von 7000' Höhe jenseits der Ebene Metidjah, wohin ich auch noch zu gehen gedachte. Der eben beschriebene näher bei Algier gelegene Theil der Sahelberge gewährt eine Ansicht ähnlich der von Nizza, und ist wohl der am meisten bevölkerte Theil von Algerien.

Hier sieht man eine Menge von weissschimmernden Landhäusern von der üppigsten Vegetation, von blühenden Sträuchern, immer grünen Bäumen, von Johannisbrod-Fruchtbäumen und hohen Cypressen umgeben, über alle aber erhaben, die einzeln stehende königliche Palme, deren schlanke Blätterwedel sich schon von fern vom blauen Himmel abheben. Dort liegen die Schlösser oder Landsitze einiger vornehmen

*) Neue Briefe aus Algerien. Brandeuburg 1858 bei J. Wiesike. 
Mauren, die des Gonverneurs, des Generals Jusuf, ein Frauenkloster, so wie die Dörfer: Mustapha en bas und superieure, Birmandrais, d'Hussein-Dei, Kouba, der Gouvernements - Garten (Jardin d' essai) Maison-carrée und mehrere andere Ortschaften, zum Theil südlicher in den Thälern des Sahel versteckt. Den obern Theil der Stadt krönte früherhin die Residenz des letzten Dey oder die theilweise zerstörle Casbah; doch liegt jetzt noch höher hinaufgebaut, das die Gegend beherrschende Fort l'Empereure, welches ich seiner schönen Aussicht wegen einige Male besuchte.

Auf diese beraseten Felsen hinauf, so wie auf allen unsern Ausflügen fanden wir eine nicht gezählte Menge blühender Sträucher und Pflanzen, von welchen ich nur Haidekraut, Cytisus, Viburnum, Orchideen und allerliebste Irisarten nennen will, zu denen sich riesige, obwohl noch nicht blühende Exemplare von Opuntia Ficus indica und Agave americana gesellten.

Zu den besuchtesten Promenaden in der Nähe der Stadt gehört eine der schönsten hiesigen Anlagen, nämlich der öffentliche Garten "Marengo" mit der Aussicht aufs Meer und geziert durch die kollossale Marmorbüste Napoleon I. Der seltenen Baum- und Strauch-Arten, so wie der Zierpflanzen aus wärmeren Himmelsstrichen sind hier eine zahllose Menge, und die einheimisch gewordene Cactusart, Opuntia Ficus indica Mill. erreicht in dieser günstig gelegenen Localität in dem vielarmig verzweigten baumartigen Stamme eine Stärke von 1 Fuss im Durchmesser.

Die sonntäglichen Landpartien, welche der Prof. D ü r a n do veranstaltete, bei welchen ich eingeführt war, gehörten mit zu den angenehmsten Genüssen, welche mir därgeboten werden konnten. Irgend eine express angenommene Diligence, vereinigte 10-14 einheimische oder fremde Naturforscher, von denen einige Schmellerlinge jagten, andere Käfer sammelten, die meisten aber die selteneren Schătze der Flora in ihre Mappen oder Kapseln bargen. Bald war unser Ziel die sogenannte Boujarẻah oder der höhêre Theil des Sahelgebirges bei St. Eugène, westlich von Algier nach Coléah zu, bei welcher Stadt die Myrtengebüsche zu Hause gehören; bald führte uns unser Prässes in die entgegengesetzte Richtung nach Maison carrée, wo $\mathbf{4 0 0}$ arabische Strafgefangene die Blätter der Zwergpalme, Chamerops humilis L., welche hier ein grosses Culturhinderniss bildet, in feine Fasern aushecheln. Diese werden zu Stricken, MaIrazzen, Matten und hundert anderen Dingen verarbeitet, ja Herr F o u c o $1 \mathrm{t}$ in Birmandrais fabricirt alle gangbaren Papiersorten daraus, und versendet die Masse selbst bis Hamburg.

Eine andere, an und für sich höchst interessante Excursion nach dem ebenfalls östlich von Algier am Meere gelegenen Jardin d'essai (Versuchsgarten) wurde durch den eintretenden Gewilterregen unterbrochen. Dort werden vom Gouvernement nützliche oder seltene Bäume, Sträucher und Pflanzen angebauet, unter welchen die Dattelpalme, welche jedoch nur in der Wüste vollkommen reife Früchte trägt, das Bambusrohr, die Banane, die Artischocke, die Cochenillen- 
pflanze, Opuntia coccinellifera Mi Il. nicht fehlen. Ausgedehnte Gewächshäuser bergen eine Menge seltener Pflanzen.

Ein dort angestellt gewesener, jetzt aber nach Bona versetzter Beamter, Dagon de la Conterie, den ich gelegentlich bei seiner Uebersiedlung dorhin auf einer Seereise nach Stora kennen lernte, theilte mir als interessante Notiz mit, dass die Kultur der Cochenille sehr mühsam, dagegen der Versuch mit einer Seidenraupe, die sich von dem Laube des Wunderbaumes, Ricinus communis L. nübre, und ein sehr werthvolles Gespinnst liefere, als vollkommen günstig zu betrachten sei. Der Wunderbaum, den man in unsern Gärten als Zierstaude sieht, wächst in der Gegend von Algier häufig wild, und hat die Grösse unserer Hollundersträucher.

Auf diese Weise und vermittelst sonstiger Spaziergänge lernte ich die Umgegend von Algier ziemlich genau kennen, und der erste zauberische Eindruck, den dieselbe auf mich machte, hat sich während meines fünfwochentlichen Aufentbaltes daselbst immer gleich frisch erhalten.

So sammelten wir auf einer der erwähnten gemeinschaftlichen Excursionen nach Coléah zu am 21. März vorlängs des Meeres, an der hügelichen Kunststrasse, so wie an Hecken, folgende Pflanzen ein :

Bellis annua $\mathrm{L}$ überaus gemein auf Rasen; Lycium europaeum L., Viburnum Tinus L. baumarlig in Hecken; Erodium chium W. an feuchten Strassenmauern; Aristolochia altissima D e sf. Klettenstrauch in einer Hecke; Aristolochia longa L., Parietaria mauritanica Duri e , Rumex bucephalophorus L. auf Aeckern neben der Chaussée; Succowia balearica M edic. und Trifolium tomentosum L. auf Steingerölle.

Eine der angenehmsten und ergiebigsten Fahrten machten wir am 28. März nach Maison carrée, wo theils auf niedrigen grünberaseten Hügeln, theils an den flachen Ufern eines weiter nördlich in das Meer fallenden Flüsschens - l'Harratch - nachstehende Seltenheiten aufgenommen wurden: Astragalus caprinus L., Borrago longïfolia $\mathrm{P}$ o ir., Coriaria myrtifolia L., Hedisarum capicatum D es f., Matope malaccides L., Denanthe globulosa L. in einem kleinen Sumpfe, welche letztere eine 6-10-strahlige Dolde hat. Die Fruchtdöldchen sind kugelig, die sitzenden Früchte gross. Die Wurzelblätter doppelt gefiedert, haben 1-2" lange schmal-lineale Fiedern. Besonders lieb waren mir die prächtige Orchis papilionacea $\mathrm{L}$. auf einem Hügel neben der schon leider längst verblühten 0 . Robertiana L o i s. und die wunderschöne Ophrys Speculum L k., Pflanzen, die ich hier zum Erstenmale lebend erblickte. Dann: Orobus atropurpureus De sf, Osyris alba L., Ranunculus macrophyllus $\mathrm{L}$ e d e b. an einem Wassergraben. Dieser Ranunkel stellt eine hohe ansehnliche Pflanze dar. Die gelappten rundlichen Wurzelblätter sind sehr lang gestielt; anliegend und der Stengel hingegen abstehend behaart, die oberen Blätter 3-theilig, ganzrandig; die Blumen gross, goldgelb; der angedrückte Kelch langzottig; der Griffel stark hakig gebogen. Ta- 
marix africana D e s. oder gallica L. war nicht zu bestimmen, weil sich nur unaufgeschlossene Blüthentrauben ohne Blätter zeigten. Zuletzt theilte mir ein pariser Naturforscher noch eines von seinen wenigen Exemplaren der Euphrasia latifolia $\mathrm{L}$. (Trixago $\mathrm{R} \mathrm{ch}$ b.) mit.

Bei einer früheren Gelegenheit, wo das Innere des arabischen Strafgefängnisses besichtigt wurde, hatten diese Lokalitälen schon Folgendes dargeboten :

Pyrethrum fuscatum W., Anemone palmata L., Helichrysum Stoechas Mn ch. unter Gesträuch apf Thonboden, wogegen Cynoclossum pictum A it. und Ophrys lutea $\mathrm{C}$ a v, eine bei Algier und auch bei Philippeville gemeine Orchidee, hei Maison carrée in Menge auf kalkhalligen Hügeln standen. Tetragonolobus purpureus $\mathrm{Mn} \mathrm{ch}$. schmückle den saftigen Rasen in selir grosser Menge.

Sonst wurden von mir noch in der nähern Nachbarschaft $\mathrm{Al}$ giers, theils anf dem Sahelgebirge - Kalk -, theils in Schluchten, auf fruchtbaren Aeckern, so wie am kiesigen Meeresstrande bis zum 30. März beobachtet, oder theilweise eingesammelt, und zwar:

A. Im Sahelgebirge auf Felsen, an Abhängen, oder in Schluchten :

Arisarum vulgare Ta $\mathrm{r}$ g., Biscutella raphanifolia $\mathrm{P}$ o i. r., $C y-$ tisus spinosus La m. und C. triflorus l' H e r it., Prunus insititia L., Galium saccharatum L., Lamarckia aurea M n c h., welches schöne Gras bereils am 27. Februar oben beim Fort l' Empereur zu blühen anfing. Moraea Sisyrinchium K er. zierte hier so wie späterhin bei Philippeville alle felsigen Anhöhen. Paronychia argentea $\mathrm{L}$ a $\mathrm{m}$, Passerina hirsuta L., Trichonema Bulbocodium K er., Asteriscus maritimus M n ch., Koniga maritima R. B r., Cardamine hirsuta L., letztere an einer Hecke; Cynoglossum cheirifolium L., Globularia Alipum L., Iris unguicularis B rot. (I. stylosa Desf.) bei Maison carrée und in der Baujaréah unter Gesträuch, in welcher letztern Oertlichkeit auch Lepidium glastifolium De sf., so wie Lonicera implexa A it. vorkamen. Rosmarinus officinalis $\mathrm{L}$. ist nur beim Dorfe Birmandrais verwildert und kommt sonst in der Gegend von Algier gar nicht vor Micromeria inodora Benth. stand jedoch daselbst in grosser Menge. Ophrys fusca Lk., O. tenthredinifera W., so wie Orchis longicornu Poir. bewolınen dort so wie bei Maison carrée und Kouba die lichten Gebüsche. Von Serapias psendo-cordigera Moric. sah ich kurz vor meiner Abreise nur ein einziges blühendes Exemplar in der Gegend von St. Eugéne hinter dem. Kirchhofe an einer Schlucht.

Höher hinaufsteigend, finden sich Olea europaea L., Pinus halepensis M ill., Quercus coccifera M ill., Q. Ilex L. als Waldbäume oder verkrüppelt, und darunter Juniperus Oxycedrus L., Erica arborea L. und Artemisia arborescens L., letztere erst mit Blüthenknospen. Auf dem oberen Theil des Sahelgebirges fand ich auf dürrem Felsgrunde Vaillantia muralis L. (Galium vexans $\mathrm{R}$ e i $\mathrm{chb}$. fil in den Icon.) nicht $V$. hispida $L$. wie mir dieses $5-8^{\prime \prime \prime}$ hohe Pflänzchen in Algier genannt wurde. Denn sowohl die Stengel als die Blattflächen sind kahl; freilich sind noch keine Früchte vorhanden. Ferner 
standen auf Felsgerölle : Andropogon hirtus L, Helianthemum glutinosum Pers., Micromeria nervosa B e $\mathrm{n} t \mathrm{~h}$., Phagnalon saxatile C a s S., so wie zwischen Felsblöcken im Sahel, aber auch auf ebenem Boden bei Maison carrée Ranunculus flabellatus D es f. Oben beim Kaiser-Fort entdeckte ich die hier keinesweges gemeine Anchusa lanata L.

In Betreff der sehr elegant aussehenden Orchis longicornu P o ir. möchte ich hier noch bemerken, dass deren Sporn sehrlang und am Ende keulig verdickt ist. Der Mittellappen der Unterlippe ist schmäler und kürzer als die fast zurückgeschlagenen und abgerundeten Seitenlappen. Diese letzteren sind ausgebissen, geründert und gewöhnlich schwarz-violett; der Mittellappen ist hellviolett mit grossen dnnkelvioletten Punkten; der Helm röthlich. Aendert wie die ähnliche $O$. Morio in der Farbe ab, und fand sich bei Kouba auch schneeweiss.

Am Rande eines Hohlweges im Sahelgebirge und zwar bei dem Dorfe Birmandrais bemerkte ich Asplenium Adianthum nigrum und A. Trichomanes L., neben welchen ich auch Selaginella helvetica Sp ring. (?) leider noch ohne Fructification, aufnahm. Von $\mathrm{M} \mathrm{u} \mathrm{m} \mathrm{b} \mathrm{y}$ ist nur Lycopodium denticulatum L. angegeben, von welchem er sagt: „commun dans les chemins maures sur les terires à l' abri du soleil. En hiver." Das Ptlänzchen scheint mir etwas derber wie unsere Saginella; doch finde ich die Bläller nicht merklicher gesägt, wie meine anderen Exemplare, und namentlich wie eines aus der Gegend von Botzen, welches ich von Herrn Sch o $\mathrm{g}$ er erhielt.

Calendula arvensis L. und Centaurea pullata L., dagegen zeigte sich Calendula officinalis L. nur an Einer kleinen Stelle nach dem Kaiser-Fort zu. Asphodelus ramosus L. sieht man in Algerien auf Hügeln und heraseten Felsen in grosser Menge, und Pistacia Lentiscus L. ist ein überaus gemeiner Strauch; Arum italicum L. blühte diesseits des Jardin d'essai und beim Dorfe Mustapha supérieur an Hecken, woselbst auch Rosa sempervirens L. bereits am 28. Febr. entwickelt war. Dann Fedia cornucopiae D e c., Fumaria agraria L a j. und capreolata L., Lupinus angustifolius L. stand zerstreut in der Boujaréah, häufiger am Wege und auf Aeckern in der Ebene Metidjah. Ferner : Salvia Verbenacea $\mathrm{R} \mathrm{chb}$. Icon. var. multifida stieg bis zum trocknen Felsen bei Maison carrée hinauf, war jedoch nur sparsam vertreten. Eben so Silene lusitanica L. selten; $S$. fuscata Lk. wuchs nur an einem Grabenrande bei Kouba, wogegen S. gallica L. gegen Ende März auf den meisten beraseten Hügeln in der Nähe der Stadt massenhaft zu finden war. Letztere ist in Re i ch b. Icon. Band VI. Tafel CCLXXII, Fig. 5054 sehr gut dargestellt. Der einfache Stengel wird fusshoch; die Blüthen sind klein, blass röthlich, stehen immer einseitswendig und sind mit langen weissen Zottenhaaren bekleidet. Dem Habitus nach ist diese Pflanze durchaus abweichend von unserer norddeutschen S. silvesiris S ch o It. Auch Allium triquetrum 
und Cerinthe major L. var. purpurascens machten sich an Hecken und trockenen Gräben gánz besonders durch ihre Menge bemerkbar. Vinca major L. und die prächlige Anemone coronaria L., die jenseits des Meeres, in der Gegend von Nizza, alle Olivenhaine schmückt, war hier ausnehmend sparsam vertreten. Auch Prasium majus L., Ruscus Hypophyllum L. blühend und mit Beeren, so wie Tamus communis L. kamen mir nur hin und wieder in Hecken zu Gesicht. Weniger selten sind Ferula sulcata D e s f., erst Ende März einzelne blühende Dolden zeigend, so wie Barkhausia taraxacifolia D ec., Smyrnium Olusatrum L. und Hyoseris radiata $L$. Unweit der Casbah auf beraseten Hügeln, oder an feuchtsandigen Stellen schlossen sich in den letzten Tagen meiner Anwesenheit in Algier immer mehr und mehr die jüngern Bürger der dortigen Flora auf, und es traten noch Plantago Serraria L., P. Psyllium L., Euphorbia verrucosa L. (?), natürlich noch ohne Kapseln und desshalb nicht sicher zu bestimmen; Convolvulus altheoides L. var. hirsutus, so wie Andryala sinuata L. in Blüthe. Auffallend ist, dass die bei uns so weit verbreitete Gattung Hieracium in Algerien ganz zu fehlen scheint. An einem Bache in der Boujaréah hatte sich ausser Scrophularia sambucifolia L. noch eine verwandte, mir aber unbekannte Art, welche Herr Dü ra ndo Sc. laevigata $\mathrm{V}$ a hI nannte, und höher hinauf in einigen WasserTümpeln beim Dorfe Boujaréah unsere gewöhnliche Form der Callitriche vernalis $\mathrm{K} \mathfrak{u} \mathrm{t} \mathrm{z}$. angesiedelt.

C. An Wegrändern, auf Aeckern fanden sich namentlich folgende, theilweise auch bei uns gemeine, Pflanzen:

Lamium amplexicaule L., Linaria reflexa D esf., Raphanus Raphanistrum L., immer weiss blühend; Senecio vulgaris L., Stellaria media V ill., Veronica arvensis, agrestis L. und V. Cymbalaria B e rt., Capsella Bursa pastoris Mn ch., Echium grandiflorum Desf. Erodium moschatum A it., Nicotiana rustica L. noch in überwinterten alten Pflanzen auf den kultivirten Aeckern blühend. Dann Poa annua L., Oxalis cernua $\mathrm{T} h \mathrm{u} \mathrm{n} \mathrm{b}$, an der Strasse nach El Biar in Menge nnd wohl Gartenflüchtling, was auch $\mathrm{Nu} \mathrm{mb}$ y glaubt; $A$ angallis coerulea S chreb. so wie Sherardia arvensis L.

D. Auf wüsten Plälzen, an Mauern, lebten: Mercurialis ambiyua L, Reseda alba L., Borrago officinalis L., Sisymbrium Irio L. Sonchus tenerrimus L. Letzterer sehr gemein und auch auf Felsen. Chrysanthemum coronarium L., Pyrethrum Myconis M n c h., Urtica membranacea Poir., Chenopodium murale L. und Sinapis geniculata D esf.

E. Ausschliessliche Bewohner des Meeresstrandes waren : Arenaria spathulata D esf., Ecbaliunt agreste R e i c hb., Echium maritimum W., Lotus creticus L., Ononis variegata L., Senecio humilis Desf., so wie Cakile maritima L. Die letzteren 5 bei Kouba und Hussein-Dei auf Sand am Meere, erstere an der entgegengesetzten Stadtseite nach St. Eugéne zu.

$\mathrm{Zu}$ jenem freundlichen Gesammtbilde Algiers und seiner Umgebungen trug freilich auch eine weitere Fahrt nach Blidah und 
Medeah bei, die ich am 22. März antrat, einem Zeilpunkte, wo mich mein junger Freund, Herr B a r tels, verliess, um über Oran nach der Insel Madeira abzureisen.

Die Diligence fuhr Mittags ab, und so sah ich denn, das überall mit Pflanzen und Sträuchern bekleidete Sahelgebirge auf der guten Kunststrasse überschreitend, jenseits des Dorfés Bircadem die bekannte Ebene Metidjah vor mir ausgebreitet. Dies ist eine baumlose Fläche sehr guten Bodens, die bei einer Breite von 3-5 Meilen eine Länge von deren $\mathbf{1 5}$ hat, und südlich vom kleinen Atlasgebirge begrenzt wird. Die diesseitige Hälfte fand ich bei Weilem geringer wie die Südseite cullivirt, weil die abdachende Hügelkette des Sahel überall mit der, von den Colonisten mit Recht so gefürchteten ZwergPalme bedeckt ist, die näher nach Blida hin auf der niedrigen Ebene gar nicht gedeiht. Anstalt ihrer sieht man dort in ungeheurer Menge den ästigen Asfodill, aus dessen knollenartiger Wurzel Spiritus gebrannt wird, so wie da, wo es niedriger war, Scilla maritima L., welche man bei uns als Zierpflanzen in Töpfen zieht.

Chamaerops humilis L. bildet auf den lehmigen niedrigeren Hügeln des Sahel mit Passerina hirsuta L., Pistacia Lentiscus L., Phyllirea latifolia L.. Cytisus-Arten u. s. w. ein dichtes Gesträuch, zwischen welchen häufig das rohrarlige Gras, Ampelodesmos tenax Lk. freudig wuchert.

Etwa in der Mitte der Melidjah, und zwar jenseits eines stundenlangen gras- und binsenreichen Sumpfes, aus welchem hin und wieder hohe dottergelbe Lilien ihr Haupt erhoben, wechselten wir in dem grossen und ansehnlichen Dorfe Boufarik die Pferde, wo europäische Colonisten und namentlich die deutschen, eine bedeutende Kultur entwickelt haben. Im verflossenen Jahre war dort für 200,000 Fr. Tabak verkauft, eine Pflanze, die in Algerien, wo keine Regie ist, gut gedeihet und rentirt. Gegen Abend erreichten wir das so hoch gefeierte Blidah, welches man einen Korb voll schönen Blumen, oder auch das Paradies Afrikas genannt hat, und welches halb in der Ebene, halb auf dem Hügellande des hier südwärts beginnenden kleinen Atlas gelegen ist. Im Hôtel de la Regence fand ich eine vortreffliche Aufnahme. Blidah ist eine kleine aber ungemein freundliche Stadt, mit geraden Strassen, guten Häusern und mehreren geräumigen Plälzen. Sie hat Ueberfluss an Wasser, und nur diesem Umstande, nicht elwa ihrer wärmern Lage wegen, verdankt sie die ausgedehnten Orangenund Citronen-Wälder, die sich stundenweit erstrecken. Ausserdem sieht man Feigen-, Granat- und Johannisbrod-Bäume, Ceratonia siliqua L., unsere Obstarten und einzelne gewallig hohe Cypressen.

Die mit weiss oder röthlich blühenden Obstbäumen bedeckten Gärten, die zierlichen Landhäuser, oder die zwischen Orangen und Feigen halbverborgenen Colonen-Wohnungen umgeben die Stadt von allen Seiten. Leider blühten erst einzelne Orangen-Bäume, wogegen andere, so hoch wie unsere höchsten Apfel- oder Birnbäume, noch reichlich mit Früchten pranglen. Die Araber stutzen nämlich ihre 
Bäume nicht, wogegen es die Europäer für zweckmässiger, halten, zu thun.

Am folgenden Tage fuhr ich mit der eben abgehenden Diligence nach Medeah, einer Stadt im kleinen Atlas gelegen, die man schon der pittoresken Strasse und ihrer Lage wegen (angeblich fast $\mathbf{3 0 0 0}^{\prime}$ ) gern besucht.

Der Weg führt zuerst durch die Ebene am Rande der Metidjah das Gebirge links lassend, bis zum Hervorbrechen der überall mit Oleander besäumten Gewässer der Schiffa aus den Vorbergen des kleinen Atlas. Diesen letatern ersteigt man sodann, das schauerlich tiefe Flussbett der Schiffa zur Linken, auf einem dem Gebirge $a b-$ gewonnenen zwar guten, doch só schmalen Wege, dass nur eben Raum genug für die zu vieren breit bespannten Pferde bleibt. Das Allasgebirge war hier überall mit blühenden Sträuchern und immergrünen Eichen bewachsen, und von den rechis ansteigenden Felsenmassen rieselten an manchen Stellen kleine Cascaden über die Strasse, die Wassermasse der jetzt kleinen Schiffa vermehrend.

Am lebhaftesten schien mir die Vegetation in der Nähe der Station Ruisseau des singes, wo ein grösserer Bach aus einer stark beholzten Gebirgsschlucht herniederbrauset, und einen Lieblingsaufenthalt des hier vorkommenden Affengeschlechts, Macacus ecaudatus (P it h è que, Cuvi e r) abgibt.

In dieser Gegend sah man die mannigfaltigsten Gewächse, hier wucherte an nassen Felsenwänden das niedlichste unserer Farrenkräuter, das Frauenhaar, Adiantum Capillus Veneris L. am häufigsten, hier blühelen gelbe Ginsterarten, so wie Erica arborea am üppigsten.

Höher hinauf starrten uns nackte Felsen entgegen; denn wir hatten das tief ausgehöhlte Bett der Schiffa verlassen, welche man vermittelst steinerner Brücken öfters überschreitet. In den links oder rechts sich öffnenden Srhluchten bemerkten wir niedrige arabische Lehmhïtten, deren Bewohner etwas Ackerbau treiben, und endlich trat uns das hohe Gebirgs-Plateau klar entgegen, auf welchem die Stadt Medeah liegt. Wir Männer stiegen zu Fuss hinauf, und entdeckten nach einem beschwerlichen Marsche unser Reiseziel, umgeben von hohen in Blüthe stehenden Mandeln- und Aprikosen-Bäumen, in einer flachen Einsattelung des Plateaus. Ich trat des Abends um 6 Uhr in Gastronome, dem besten Gasthofe, ab.

Die Lage der Stadt ist, wie bemerkt, eine hohe; doch sieht man auch hier, wie in der Nähe aller Städte Algeriens, einen mit Fleiss betriebenen Ackerbau. Vorzüglich blühend schien mir derselbe vor dem südlichen 'Thore, wo eine tiefe Schlucht beginnt, die die Stadtmauer zum grossen Theil umgibt. Indem ich hier am 24. März früh die Gemüsegärten vor dem Thore des Jardins und die wieder höher hinauf liegenden schönen Weizen- und Bohnen-Felder durchwanderte, um die mit Wein bepflanzlen Hügel aufzusuchen, zogen einige entferntere Kuppen des Atlas meine Aufmerksamkeit auf sich, und ich beschloss, meine kleine botanische Entdeckungsreise dorthin $\mathrm{zu}$ richlen. 
Ein Hügel nach dem andern wurde überstiegen, ein kleines quellenreiches Thal un das andere durchwandert, bis ich mich in einer tiefen Schlucht befand, wo mir äusserst bissige arabische Hunde die Passage streitig zu machen suchten. Ihr Herr jedoch, aus seinem Lehmhanse herbeieilend, befreite mich von ihnen, und so konnte ich meinen Weg bis zum Fusse der höheren Gebirgsriesen fortsetzen, wo ein breiter sehr schnell fliessender Bach, vielleicht zum Quellengebiet des Bou Roumi gehörend, meinen weilern Forschungen eine Gränze setzte. Seinem Laufe folgend, traf ich in dieser nach und nach öde gewordenen Gegend, wo ausser einer seltenen Orchidee, Orchis patens D e S f? nur Ginster, Wachholder und verkrüppelte immergrüne Eichen standen, später wieder auf einige Spuren menschlichen Wohnsitzes, nämlich auf verwilderte Mandelbäume, in deren Nähe auch in der That eine kleine Wassermühle lag. Der freundliche Besitzer nannte den Mühlbach „Barrora“.

Als bolanische Ausbeute brachte ich noch Alyssum atlanticum Desf., Coronilla pentaphylla D esf., Saxifraga granulata L, eine in Algerien seltene Pflanze; Bellis sylvestris C y r., letztere anf quelligem Moorboden, und vom Ufer jenes Gebirgsbaches ein Medicago ohne Hülsen, aber wahrscheinlich M. lupulina L., so wie Salix pedicellata D e sf. mit zur Stadt zurück. Vor dem Thore standen noch hart am Wege auf Rasen: Ranunoulus spicatus $\mathrm{D}$ e $\mathrm{s} f$. neben Silene rubella L., so wie in den offenen Gemüsegärlen mehrere Formen unserer Fumaria officinalis $\mathrm{L}$. Alyssum atlanticum $\mathrm{D}$ es $\mathrm{f}$. ist unserem A. calycinum L. zu vergleichen, aber mit grösseren Schötchen, die oben deutlich ausgerandet sind. Meine Exemplare standen auf dürrem Boden, haben nur eine Höhe von $\mathbf{2 - 4}$ ", eine dottergelbe Blüthenfarbe, einen einfachen oder verästelten Stengel und sind überall sternförmig behaart.

Die hier bei Medeah gefundene Orchidee ist eine zwoifelhafte Pflanze, wohl in der Formenreihe von Desfontain es O.patens gehörig, aber wegen der Lange des Sporns nicht die von ihm beschriebene Species genau. Sie macht den Eindruck einer kleinen o. mascula L., welche aber, abgesehen von anderen wesentlichen Abweichungen, selbst in der wärmeren Gegend von Maison carrée viel später, nämlich im April und Mai blüht. Auch wurde diese Pflanze im frischen Zustande von Herrn Dü rand o sofort als 0 . patens De s f, erkannt, und anderen gerade anwesenden Botanihern als Seltenheit angezeigt.

Die Knollen dieser 6-8" hohen Orchidee sind oval, mit wenigen fadenförmigen Nebenwurzein. Die 5-8 wurzelständigen, ungefleckten Blätter oberhalb der Scheide verschmälert, sind länglich, nicht breit, und kurz zugespizt. Ueber denselben befinden sich 1-2 scheidenartige kleinere. Die Blüthentraube ist kurz, 11/2-2", aber weit, tockerblülhig, 6 - höchstens 10 blumig. Die häutigen, und wie der obere Theil des Stengels röthlich angelaufenen Deckblätter sind lanzettlich, kürzer als der gedrehte Fruchtknoten; die aufrecht abstehenden Blüthchen lebhaft purpurroth. Die Lippe ist am Grunde 
keilförmig, 3-lappig, stark geadert; der etwas vorgezogene und tief ausgerandete Mittellappen trägt gemeiniglich in der Ausrandung einen hleinen Zahn ; die Seitenlappen, so breit als der mittlere, sind bogenförmig gekrïmmt und unregelmässig gekerbt. Der gekrümmte, cylindrische Sporn ist abstehend oder aufsteigend, und so lang oder sehr wenig kürzer, als der Fruchtknoten, zur Zeit der yollkommen aufgeschlossenen Blumen.

Uebrigens ist hier noch anzuführen, dass in der Höhe von Medeah die schwer auszurottende Zwergpalme gänzlich fehlt, deren Stelle aber auf dem rohen unbearbeiteten Lande Ginstergewächse und Eichengestrüpp einnehmen.

Von einem Bekannten, dem Herrn Notar La u ja u let, halte ich einen Empfehlungsbrief an den Unterpräfecten, Hrn. C h a n c el in Blidah, erhalten, den ich bei meiner Rückkehr von Medeah abgab. Der Herr S ous-Préfet, ein sehr unterrichleter und wohlwollender Mann, führte mich auch nach seinem Garten, auf dessen Pflege derselbe viel Sorgfalt zu verwenden schien. Sie hatte auch Erfolg gehabl. Die Vegelation war eine üppige, der schnelle Wuchs der kïrzlich angepflanzten Bäume und Ziersträucher bewundernswürdig, wogegen die Bananen nicht gedeihen wollten, die in und beim Jardin d' essai bei Algier so schön standen.

Weiterhin musste mich Mustapha, sein Araber, hinaus in die Ilerrlichkeit dieser Orangenwälder und Olivenhaine führen, von wo uns aber ein unbequemer, wenngleich warmer Regen zu Hause scheuchte. Dennoch hatten wir mehrere Landhäuser besucht, die zwischen blühenden Bäumen und hinter den einzeln stehenden Johannisbrot-Bäumen oder Palmen hervorblickten, und in der Nähe eines neu angelegten Kirchhofes eine mächtige Pinie aufgesucht, die in einer Höhe von $3^{\prime}$ über der Erde ein en Umfang von $1^{\prime}$ hatte. Merkwürdig contrastirten gegen das saltgrüne Laub jener Hesperideen die lichtgrünen hängenden Blätterzweige der Thränenweiden, die man in Algerien so häufig angepflanzt sieht.

Blidah war also sehr schön, eingehüllt in Grün und Blüthenschmuck; will man es jedoch im höchsten Flor sehen, dann muss man es 14 Tage später, wie ich es vermochte, besuchen, mithin elwa Mitte April, wo Orangen- so wie Oel-Bäume ihre Wohlgerüche aushauchen.

Mustapha halte mich bei dieser Gelegenheit auch nach dem Fusse des kleinen Atlas in eine Schlucht begleitet, an deren südlichem Rande sich nicht nur ein Marabut befand, sondern die auch von einem Gebirgsbache, El-Kebir, durchrauscht wird, dessen Gewässer weiter unten den Orangengärten zugeführt werden. In dieser Schlucht, mithin in der Năhe des Oued-El-Kebir, hatle ich noch Musse genug, einige interessantere Pflänzchen als : Lysimachia Linum-stellatum L., Plantago microcephala $\mathrm{P}$ o ir. und Silene pseudoatocion D e s f. mitzunehmen, welchen späterhin noch Galactites tomentosa $\mathrm{M} \mathrm{n} \mathrm{ch}$. neben den arabischen Mühlen, Ranunculus muri- 
catus $\mathrm{L}$. an Bewässerungsgräben, so wie Linaria virgata $\mathrm{D}$ e $\mathrm{s}$. vom ehemaligen Kirchhofe zugesellt wurden.

Am 3. April verliess ich Algier, und fuhr mittelst Dampfbootes über Stora nach Philippeville, so wie von dort mit der Diligence nach Constantine, wo wir - ich mit einigen befreundeten Reisegefährten - wohibehalten am 6. des Morgens früh eintrafen, Wir fultren jedoch schon am folgenden Tage mit der kleinen Diligence zeitig weiter nach Batna, um uns von da aus dem Rande der Wüste zu nähern.

Wahrend der ersten Hälfte des Weges waren die Ländereien gut angebaut und hatten viel Weizenfelder aufzuweisen; nur Schade, dass diese Frucht nicht rein, sondern von der wilden Artischocke, Cynara Cardunculus L, sehr unterdrückt war. Dieses distelartige Gewächs, welches man auf allen Feldern bei Constantine in Menge sieht, wird zwar von den Arabern gegessen, ist jedoch keine geschälzte Marktwaare. Dagegen fehlt hier zum Glück für den Ackerban die gefährliche $Z_{w}$ ergpalme gänzlich, wenigstens habe ich deren nicht gesehen.

Des Mittags wurde in einem einzeln liegenden Gehöft, eine Art Karavanserei, gegessen, und dann die ziemlich langweilig werdende Reise fortgeselzl. Denn wir kamen nur durch wüste unbebauete Gegenden, wo wir nichts als wandernde Araber in schwarzbraunen Zelten sahen, die dort ihre Schaf-, Ziegen- und KameelHeerden weideten, welche letzteren 50-200 Häupter enthielten. Nur diese Fremdlinge hielten unsere Aufmerksamkeit -wach, weil wir sonst, mit Ausnahme eines todten Salzsees, nur hügeliges Land sahen, und die nähern Gebirgsketten unbewaldet fanden. Wir haben in Wirklichkeit von Constantine bis Batna, auf einer Strecke von wenigstens 24 Lieues, nur kurzes Gesträuch und keinen einzigen Baum gesehen. Jenen Salzsee aber, deren es in dieser Gegend mehrere gibt, nenne ich deshalb todt, weil er von keinen Schwimmvögeln belebt, und der sandige Strand ohme irgend eine sichtbare Spur von Pflanzenwuchs war. Die Vegetation auf den von uns überblickten Flächen zeichnele sich zwischen Constantine und Batna überhaupt weniger durch Reichthum der Pflanzenformen, als durch ein massenhaftes Auftreten einzelner Arten aus. So dominirte südlich von ersterer Stadt die bereits erwähnte wilde Artischocke, wogegen in der Gegend des Salzsees, mithin etwa in der Mitte zwischen beiden Ortschaften, auf allen wüsten Ländereien die gelbblühende Othonna cheirifolia L. vorherrschte, oder die niedlıche Levkoje, Matthiola lunata $\mathrm{D}$ ec. die Erde mit ihrem Violett bedeckte. Beilüufig bemerke ich, dass diese Athonna nicht glatte, sondern filzige-, mit einer langen Haarkrone gezierte, Achenen hat. Der Kelchabschnitte sind 8, der Blumenblätter 16-18 vorhanden.

Von Batna führt eine gate Kunststrasse den Reisenden durch die Ebene nach dem eine Stunde entfernten Lambessa, wo ich am folgenden Tage neben den Trümmerhaufen der ehemaligen Hauplstadt Numidiens von dem dürren steinigen Boden einige Exemplare von 
Anacyclus Pyrethrum D e c. und Astragalus tumidus W. (Anthyllis tragacanthoides D esf.) zur Erinnerung mitnahm. Auf dem ebenen Felde bei Balna aber erfreule mich eine dort vereinsammt am Wege stehende Tulipa Celsiana D e c., die meinem Herbare noch abging.

Um von Balna nach Biskara - auch Biskra - zu gelangen, muss man, obwohl der Weg bis zur nächsten Karavanserai Luxor noch ziemlich gut ist, entweder reiten, oder das einzige Fuhrwerk zu benutzen suchen, mit welchem der Lohnkutscher Hippolyte dann die Reise nach der genannten Oase unternimmt, wenn sich eine lohnende Gesellschaft findet. Dies war nun gegenwärtig der Fall, und so verliessen wir un 6 Uhr früh Batna, bei schönem beitern Wetter.

Wir hatten zu beiden Seiten hohe bewaldete Gebirgszüge, zwischen welchen ein Hügelland befindlich ist, das bis zur Karavanserai Luxor einen ziemlich ebenen Weg darbot, auch hin und wieder cultivirt war. Die Karavanserai, wo wir des Mittags ankamen, und zwei Stunden blieben, liegt auf freiem Felde dicht neben einem Keinen Bache, wo eine Araber-Familie ihre Esel mit den eben gefüllten Wasserschläuchen belastete. Dieser Bach, welcher im nahen Gebirge seine Quellen hat, tränkt bei El Kantara schon die Palmengärten, und wird dann, immer weiter südlich fliessend, zum Oued (Uüd) Bìskara, der sich späterhin in der Wüste verliert.

Bei der Karavanserai Luxor bolen die näheren Umgebungen des genannten Baches nur eine ziemlich dürftige Vegetation und zwar Nachstehendes dar: Ceratocephalus falcatus Pe rs., Plantago ovata F o rsk. Letztere ist nur 1-2" hoch, bewollt, vielstengelig; die Köpfchen einzeln, rundlich. Dann Hypecoum pendulum L., Micropus supinus L.; ein sehr niedriger, dottergelb blühender Steinklee, vielleicht Melilotus sulcata $\mathrm{D}$ e $\mathrm{s} \mathrm{f}$., ist wegen Mangel der Hülsen nicht sicher zu bestimmen, und Adonis flammea $\mathrm{J}$ a c q., wofür ihn mein junger botanischer Freund, Herr Dr. P. A s c h e $r$ in Berlin, ganz richtig erkannte. In Algerien passirte diese Art deshalb schon mit Unrecht für den allerdings sehr ähnlichen $A$. aestivalis L., weil die Kelche behaart und die Blumenblätter oben an der Spitze gezähnt sind; Früchte zeigten sich noch nicht. Diese kleinen Pfiänzchen vegetirten auf trockenem lehmigen Boden, wogegen Potamogeton densus L. blühend und mit ausgebildeten Nüssen in dem Bache fluthete. Der Lauf des Letzteren und die denselben bezeichnenden Oleandergebüsche deuteten uns in Allgemeinen den Thalweg an, welchen wir bis zur gedachten Oase zu verfolgen hatten.

Von hier an wurde die Gegend raub und öde. Der Boden halte viel Einrisse, war steinig und baumlos, und nur die höheren Gebirge, die uns östlich blieben, waren beholat. Wir folgten der eben nur sichtbaren Strasse über Berg und Thal, indem wir im Verlaufe der Zeit den Gebirgsbach wohl 10-12-mal durchkreuzten. Hier und dort bleichten Gerippe von Kameelen, und auf diesen dürren steinigen Hügeln, ohne sichtbare Spuren von Leben, kamen wir uns ganz verlassen vor. Vor uns aber erhob sich eine andere, von Westen 
nach Osten streichende nackte Gebirgskette, die den Weg zu versperren schien. Es war das El Kantara-Gebirge, welches uns durch den wunderbar schönen Anblick der Gebirgsschlucht überraschte, durch welche der hier schon breite, schnell strömende Bach seinen weitern Lauf erzwungen hat. Eine Römerbrücke, deren Ecksteine noch sichtbare Inschriften tragen, führte über dieses Gewässer und eröffnete uns die lachende Aussicht auf den Palmenwald von El Kantara, neben welchem die aus niedrigen Lehmhäusern bestehende AraberStadt liegt.

Am kommenden Morgen, abermals dem Laufe des Wassers folgend, hallen wir links vor uns rothe Sandsteingruppen, rechts noch eine geraume Zeit die Palmengärten El Kantaras, welche auch viele im frischesten Lichtgrün prangende Feigenbäume bargen. Dann noch einige Streifen mit Gerste, die bewässert werden konnten, und zuletzt wieder denselben abscheuligen Weg wie gestern. Nur eine Stelle, eine langgedehnte quellenreiche Niederung, machte eine Ausnahme. Hier war der Boden, worauf hin und wieder Binsen wuchsen, mit einer schneeweissen Kruste bedeckt, die wir für Salz hielten. Gegen 11 Uhr früh erreichten wir die Karavanserai El Outaja, in deren Nähe das ziemlich grosse Araber-Dorf mit seinen Gerstfeldern und Gärten liegt, aus welchen letztern einige Palmen hervorragten.

Die Ebene, welche kurz darauf vor uns lag, war unabsehbar wie die Metidjah, und baumlos, wie diese. Links und rechts hatten wir im Anfange abgeerntete Baumwollenfelder, welche das Gouvernement durch ein Militär-Commando unter der Aufsicht eines Gärtners bebauen lässt, welches Personal in der Karavanserai untergebracht ist.

Die Baumwollenstaude, Gossypium herbaceum L. gedeihet nach der Versicherung des Gärtners auf diesem kalkhaltigen Lehmboden sehr gut.

Jenseits dieser Baumwollenfelder sahen wir noch einige Streifen mit Gerste, dann aber nur unbebaute lehmig-kalkige Flächen, oder kiesige Riesel, die entweder ausgetrocknet, oder dürftig mit Wasser versehen waren. Auf dieser fast unabsehbaren Ebene, von Salsolaceen - Atriplex Halimus L. vorherrschend - grau überzogen, oder seltener in eine lichtere Grasniederung übergehend, weideten zahlreiche Heerden von Schafen, Ziegen und Kameelen. Oft begegneten uns wandernde Tribus, die aus dem Süden kamen, und ausser den Heerden alle ihre Habseligkeiten mit sich führten.

Zwei und eine halbe Stunde waren nöthig, um diese Ebene zu durchfahren, und dann erstiegen wir die letzten Felsenkämme, welche sich, wie die von El Kantara, der Quere nach von Westen nach Osten ziehen. Wir gingen hier zu Fuss, um den Pferden Erleichterung zil verschaffen, und so hatte ich denn Gelegenheit, noch einige hübsche Blumen, nämlich Erodium guttatum W. und Lavandula multifida L. mitzunehmen, denen weiterhin noch Sonchus spinosus $\mathrm{D}$ e c. hinzugefügt wurde. Der genannte Reiherschnabel ist ein schr zierliches Gewächs, von etwa 1'Höhe, schwach aufsteigend 
und mit feinen anliegenden weissen Haaren bekleidet; die Wurzelblätler herzförmig - länglich, unregelmässig gekerbt oder auch 3-lappig; die Lappen der kurzgestielten Stengelblätter aber 3- und mehrspaltig; die Blumenstiele sind meist 3-blüthig, die schön violetten Blumen ragen ziemlich weit über die $\mathbf{3}$-nervigen Kelchblätler hervor, und haben an ihrer Basis einen tiefschwarzen Fleck.

Oberhalb rechts sahen wir sodann einen gewöhnlichen mechanischen Telegraphen, wogegen die elektrischen Drähte von Algier über Constantine bis Batna reichen. Auf dem höchsten Punkte dieser abgeplatleten 'Tafelberge, welche nur niedrig und kaum 500 ' hoch sind, angelangt, erblicklen wir vor uns so weit der Gesichtskreis reichte, die unendliche Wüste, und vorn in derselben einen langgezogenen grünen streifen, die Oase Biskara mit ihrem Palmenwalde.

Uns ergriff bei diesem längst ersehnten Anblicke elwa dasselhe Gefühl, welches den Neuling überwälligt, wenn er zum Erstenmal den Ocean erblickt. In solchen Augenblicken gibt man seinen Empfindungen keine Worte; schweigend überlässt man sich den Eindrücken auf das Gemüth.

Die ganze Oase wird durch den bekannten von El Kantara kommenden Bach, Oued Biskara, bewässert, der in fadenartige Rinnen gespalten, überall zwischen den Palmengärten und Gerstfeldern durchgeleitet wird. Dieser Oued Biskara schwillt im Herbst und Winter, milhin in der Regenzeit, zu einem breiten Flusse an, dessen weiles Bett im Sommer, wo das Wasser zum Bedarf der Oasen abgeleilet wird, trocken und mit Kieseln bedeckt ist.

Am trockenen Flussbette standen ausser Frankenia corymbosa Desf., Dimia cordata R. Br. und andere noch nicht blühende strauchartige Gewächse. Von Herrn $\mathrm{Sch}$ m itt, Botaniker und Apotheker des dortigen Hospitals, erhielt ich einige werthvolle getrocknete Pflanzen, unter andern auch einige Exemplare der Anastatica hierochontica L., die dort am Wüstenrande vorkommt. Man rechnet bei Biskara neben 100,000 Palmen noch 20,000 Olivenbäume, ausser den vielen Feigen, Granaten und Pfirsichen.

Die Temperatur der Luft hatte sich in Folge eines Gewitters ziemlich abgekühlt. Am Abende vor unserer Ankunft in Biskara, mithin am 9. April zeigte mein Thermometer um 6 Uhr des Abends in El-Kantara noch 24 Wärmegrade nach Reaum.; nach dem Gewilter jedoch am folgenden Tage um 9 Uhr früh bei scharfem Nordwinde nur 9, $6^{\circ}+$ im Schatlen unseres Wagens. In den Monaten Juni, Juli, August ist es in Algerien am heissesten, und die Wärme steigt dann in der Oase Biskara auf $\mathbf{3 2}-\mathbf{3 8 ^ { \circ }}$ Reaum., so dass man von 8 Uhr früh bis ธ $\mathbf{U}$ r Abends nicht auszugehen pflegt. Jetzt notirte ich des Morgens bis $8 \mathrm{Uhr}$ bei ziemlich starkem Ostwinde und klarem Himmel nur $16-18^{\circ}+$ und die Wärme stieg des Mitlags $12 \mathrm{Uhr}$, wo sich der Wind vollkommen gelegt hatte, nicht über $\mathbf{2 2}^{\circ}$.

Heute, am Tage nach unserem Eintreffen, machte ich Vormillags einen Spaziergang nach dem Biskara der Araber, wohin ein schöner breiter Weg führt, von welchem ich rechts cie Palmengruppen, links 
hingegen ein Ackerfeld übersah, auf dem die reife Gerste, Hordeum hexastichon L. eben geschnitten wurde, oder es schon war. Es wuchs viel wilder, meist schon ausgefallener Hafer darunter. Den gewöhnlichen Fulterhafer, Avena sativa L. bauet man in Algerien nicht, nur aus der Gegend von Oran habe ich eine Probe gesehen. Die Pferde, auch die der Cavallerie füttert man bekanntlich nur mit Gerste.

Der Güte eines in Biskara in Bureau arabe angestellten sehr geachteten nähern Landsmannes, des Herrn Premier-Lieutenant $\mathrm{R}$ os e, verdankte ich die Benützung eines arabischen Pferdes, um unter Be gleilung eines zuverlässigen Arabers, über die Oase hinaus, einen Blick in die Wüste selbst zu werfen.

Wir passirten eines Morgens früh einen Theil der mir bereits bekannten Araberstadt, durchschnitten dann eine baum- und strauchlose Wüstenstrecke bis zur nahe gelegenen kleinen Oase Kurra, jenseits welcher ich die Sahara klar und dentlich gleich einer grossen Tafel vor mir ausgebreitet sah.

Der Boden bestand hier keineswegs aus Flugsand oder Steingerölle gleich anderen Theilen der Sahel- oder der grossen Wüste; sondern aus fester, thon- und kalkhaltiger Erde, welcher wie auch ersterem oder dem Sande nichts als hinreichende Feuchtigkeit fehlt, um überall fruchtbar zu sein. Hinter mir und seitwärts fielen die Ausläufer des Auresgebirges steil nach der Wüste ab, vor mir aber sah ich in dieser unendlich scheinenden gelbgrauen Ebene die Oase Oumach (Umasch) in einer Entfernung von etwa 4 Lieues. Etwas weiter und mehr links von Kurra schimmerle ein anderer grüner Punkt, welchen ich für Thouda hielt.

Biskara gehört mit zu demjenigen Gürtel der Wüsle am Fusse des Südabhanges des Auresgebirges, welcher reich an Oasen ist, die ihr Bestehen den vielen Bächen verdanken, welche auf jenen und den Atlas-Gebirgen entspringen und weiterhin in der Wüste versiegen. Tiefer im Süden wird eine der unerlässlichsten Bedingungen des organischen Lebens, die Feuchtigkeit, geringer; die Oasen werden seltener.

Ausserhalb der Grenzen der kleinen Oase Kurra, mithin auf dem trocknen dürren Boden, war die Vegetation beinahe Null, weil eben die Feuchligkeit fehlte, die dieses sonst anbauungsfähige Land mil Kräutern bedecken würde.

Nur nach langem Umherspähen erblickte ich einige grau aussehende Pflanzen, z. B. Salsola vermiculata L ; eine kleine Cyperoidee, späterhin vom Herrn Prof. A. B ra u in Berlin als Carew schoenoides $\mathrm{H}$ o s t. erkannt, und eine einzige ansehnlichere Blume Peganum Harmala L. schien mir, als Wüsten-Pflanze, des Mitnehmens werth.

Auf wüsten trockenen Plälzen bei Biskara selbst bemerkle ich hingegen mehrere Gräser, nämlich: Polypogon monspeliensis D es $f_{\text {, }}$, Hordeum maritimum $\mathrm{W}$ ither. Imperata arundinacea $\mathrm{C}$ y $\mathrm{r}$, so wie Phalaris canariensis $\mathrm{L}$.; aber alle vereinzelt. 
Der mich begleitende Araber sprach französisch, und ermangelte nicht, mir Alles zu zeigen, und mich überall hinzufïhren, wie seine Instruktion es vorschrieb. So hatte ich denn auch den auf $P$ ferden lewerkstelligten Transport der jetzt verpflanzbaren jungen Palmen geschen, die bereits 3-4 Jahre später Früchte tragen sollen, und die künstliche Befruchtung der weiblichen Stämme beobachten können.

Im Jardin d' essai bei Biskara, wohin mich der dortige Militärarzt, der llerr Dr. B ern a r d begleitete, werden gleichfalls Kulturversuche mit auswärtigen Gewächsen, ja selbst mit tropischen, veranstaltet. Der Direktor derselben, Herr $\mathbf{J}$ a $\mathrm{m}$ a i n, bedauerte nur, dass ich 2-3 Monate zu früh, mithin zu einer Zeit komme, welche nichts Interessanteres, als blühende Ziersträucher, Rosen u. s. w. darbiete. Von den acclimalisirten Bäumen war es namentlich der Maulbeerbaum, dessen kräftiges Gedeihen derselbe hervorhob. Hr. J a m a i n, welcher auch die Baumwollenkultur bei El Outaja leitet, hatte die Gefalligkeit, mir durch einen arabischen Gartenarbeiter die sich eben erschlossenen Blüthenscheiden beider Palmengeschlechter herabholen zu lassen. Die Dattelpalme erreicht eine Höhe von $40-60^{\prime}$, die sogar in günstiger Lage bis 'zu 30 Meter steigen soll; doch habe ich auch in arabischen Hausgärten niedrig gehaltene blühende Stämme gesehen, welche nur 6-1.' hoch waren.

Unsere Rückreise von Biskara traten wir am 13. April in derselben Richtung an, nahmen uns jedoch vor, in Batna einen Tag zu verweilen, um daselbst den sehr ausgedehnten Cedernwald zu sehen, welcher sich unweit dieser Stadt, und zwar westlich von Batna, befindet. Die Entfernang desselben von dem genannten Orte gab man selbst dort verschicden an; aber alle Welt war daruber einig, dass diese so seltenen Bäume tief im Gebirge stehen, und der Weg dahin sehr beschwerlich sei.

Meine Gefährlen wählten zu diesem Ausfluge einen schweren vierspännigen Wagen, wogegen ich einen leichten Einspänner vorzog, um höher auf das Gebirge hinaufkommen zu können. Es sind die Gebirge von Belezmah, dem Pic de Tugurt gegenüber, wo der reine Cedernbestand beginnt.

Auf der linken Abdachung des ersteren dominirten zuerst Quercus Ilex und coccifera L. mit Juniperus phoenicea L., Callitris quadrivalvis V en t. (Thuja articulata D e sf.) und daneben Erinaea pungens B o is s. (Anthyllis erinacea L.) letztere mit schönen hellblauen Blüthen. Höher hinauf, wohin meine Gefährten nicht folgen konnten, zeigte sich die Ceder, Pinus Cedrus L. in dichten Beständen vorherrschend.

Dieser, dem Gouvernement gehörige Wald war unabsehbar, und gleichwohl sagte mir der Gardegeneral, (Oberförster), welchen ich weilerhin in seiner Wolnung im Walde anfsuchte, dass sich der schönste Cedernbestand drei Stunden weiter vorfinde. Derselbe versiclrerte mir, dass er dergleichen Bäume von 25 Meter $=\mathbf{1 0 5}$ ' Höhe und 6 Meter oder 18 Fuss Unfang gesehen habe, deren Aller man auf 400 Jahre schälzen könne. Der Transport der Cedern, welcher mit Maul- 
thieren bewerkstelligt werde, sei sehr schwierig, und deswegen stello sich der Verkaufspreis pro Kubik-Meler auf 8-10 Fr.

Die von mir selbst gemessene Ceder, welche Bäume, beiläufig bemerkt, einige Aehnlichkeit mit der Rolhtanne zeigen, hatte sogar einen Umfang von $19^{\prime} 6^{\prime \prime}$. Die Rinde derselben fand ich glatt, weiss grau, die kurzen Nadeln zusammengedrückt vierseitig, lrurz zugespitzt und beim Trocknen abfällig. Der mitgenommene, noch nicht ausgewachsene Zapfen hat eine eiformige längliche Gestalt, misst

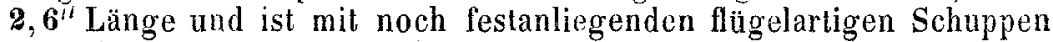
bedeckt.

Unter diesen hohen dunkeln Cedern fand ich ein kleines zartes Pfänzchen mit violelten Blühhen und kaum anselzenden Schötchen, welches, wie sich später in Berlin erwies, Arabis verna R. Br. war.

Die Ceder, vielleicht hier so kräflig, wie früher auf dem Libanon, gefällt sich in einer Höhe von $\mathbf{4 0 0 0 - 5 0 0 0 ' , ~ s t e i g t ~ a b e r ~ g e w i s s ~ n i c h t ~}$ niedriger herab, weil schon die am Thalrande stehenden, gedrúckt und verkümmert waren. Da nun die Hochebene, worauf Batna liegt, eine Höhe von mehr als $\mathbf{3 0 0 0}$ Fuss hat, und ich dem mir so nahe gewesenen, noch mit Schnee bedeckten Tugurt eine solche von 6000 bis $\mathbf{7 0 0 0}$ Fuss zuschreiben darf; so wird sich obige Annahme der Wahrheit nähern.

Die nahen hügeligen Umgebungen von Constantine und Philippeville sind, wie ich während meiner kurzen Anwesenheit daselbst fand, im Gegensatze von der kallen Hochebene bei Batna, äusserst kräuterreich. Es isl dort eine grosse Anzahl interessanter Pflanzen heimisch, von denen mehrere eine Zierde unserer Gärten sein würden. Dies beweisen nicht allein die Centurien seltener Pflanzen Algeriens, welche der Apotheker im Militär-Hospitale zu Constantine, Herr S. Choulette, herausgibt, dessen Bekannlschaft ich gelegentlich machte, sondern anschaulicher noch die Iebende Natur selbst.

Bei einem Spaziergange an der Südost-Seite dieser Stadt, welche auf einem hohen Berg-Plateau liegt, und hier vom schanerlich-lief strömenden Rummel umgeben wird, erblickte ich unweit davon einen blumenreichen Hügel, auf welchem ich $\mathbf{2 4}$ verschiedene PflanzenSpecies zahlte, die mir im frischen Zustande fast sämmtlich fremd waren. Da ich keine Botanisir-Kapsel milgenommen hatte, so gingen leider die meisten von ihnen durch Verwelken verloren. Geborgen wurden nur : Anchusa italica R etz., Helianthemum pilosum P e r's., Vicia biflora D esf., Moricandia arvensis D ec., Biscutella apula L, Linaria triphylla Mi II., Anacyclus clavatus P ers., Synapis pubescens L., Eruca sativa La m., letztere an Hohlwegen; Anagallis collina $\mathrm{S}$ c h o $\mathrm{s} \mathrm{b}$.

Die Gegend von Philippeville, und namentlich die Hügel an der Chaussee nach dem kleinen Hafen Stora, bedeckten grosse Bestände von der eben blühenden Quercus Suber L. mit unzähligen Gebüschen von Cytisus triflorus l'H erit., Cistus salvifolius und monspeliensis L. geschmückt. Sparsamer war Tolpis barbata Gaertn. so wie Myrtus communis L. Auf den niedrigeren Abhängen halten sich neben 
Anagallis latifolia L. Allium roseum L., Tetragonolobus biflorus Se r. und einige Orobanchen angesiedelt, wogegen Tctragonolobus purpureus so wie Orobus atropurpureus reichlich die Wiesengründe diesseits des Dorfes Vallée zierlen. Eben daseibst am Wegrande stand auch die 3-4' hohe Celsia cretica L., welche einem Verbascum. täuschend ähnlich sieht.

Die gelben Blumen dieser ansehrlichen Pflanze haben die Grösse jener von $V$.thapsiforme, aber von ihren 4 Staubfäden sind die beiden kürzern violett, an der Spitze gelb bewollt; die Kelchabschnitte finde ich scharf gesägt.

Am 21. April verliess ich mittelst Dampfbooles Algerien in Ge • sellschaft einiger werthen näheren Landsleule, mil denen ich mich schon in Constantine zusammengefunden hatte, und brachte in meine Heimat, ausser den sehr freundlichen und lieben Erinnerungen, eine recht liübsche Sammlung nordafrikanischer Pflanzen mit, deren grosse Mehrzahl der Flora des millelländischen Heeres angehört, und für meine hiesigen bolanischen Freunde bestimmt war.

Brand e n burg, Ende März 18.59.

Ueber den

\title{
geometrischen Charatest und über die Streckung
}

\author{
des entwickelten Laubblattes von \\ Liriodendrume tipifera.
}

\section{Von Inlius Wiesner.}

Das Wachsthum des Blattes besteht aus 3 aufeinanderfolgenden, aber von einander verschiedenen Perioden. Die erste dieser Perioden umfasst di e A n l a g e des B lat tes, wobei die Zellbildung nach abwärts statlfindet, so zwar, dass die Spitze als der erstangelegte, mithin als ältester Blattheil $\mathrm{zu}$ betrachten ist. Das angelegte Blatt ist in allen Fällen eine höcker-oder warzenförmige Excentricität, oder eine wulstförmige Erhebung an der Axe, welche durch Wachsthum (Zellbildung?) an verschiedenen, im angeleglen Blatte symetrisch vertheilten Vegelationspunkten in die Periode der Entw icklung eintritt. Das entwickelte Blatt, in welchem keine neuen Zellen mehr entstehen, keine neue Gefässbündel mehr entwickelt werden, hat sein Wachsthum noch nicht beendet, sondern tritt noch in die Periode der Streckung ein, bei welcher eine Ausdehnung der gebildeten Zellen und der Gefässbündel stattindet.

Damit das angelegte Blatt aus seiner stets äusserst einfachen Gestalt die unendlich mannigfaltigen Formen während seiner Entwicklung annehme, muss es an verschiedenen Punkten, nach verschiedenen Richtungen und mit verschiedener Intensität wachsen. $\mathrm{Ob}$ bei dieser Entwicklung an den Vegetationspunkten eine Bildung von 